\title{
Sexualidade em pacientes pós-acidente vascular encefálico vinculados a unidades de saúde na cidade de Santarém-PA
}

\author{
Sexuality in post-stroke vascular patients in Santarém-PA
}
Sexualidad en posaccidente vascular encefalico relacionados con unidades de salud en la ciudad de Santarém-PA

Yasmin da Silva Nascimento ${ }^{1}$, Jociney José Pedroso da Silva Júnior ${ }^{1}$, Rudá Ávila Monteiro', David Isaac Serruya ${ }^{1}$, Irilane de Alcântara Figueira ${ }^{1}$, Irineu Lopes de Alcântara Júnior ${ }^{1}$, Miguel Rebouças de Sousa ${ }^{1 *}$, Ruan Davi Rebouças de Sousa ${ }^{1}$, lury José Rego Moura1, Richelma de Fátima de Miranda Barbosa ${ }^{1}$.

\begin{abstract}
RESUMO
Objetivo: Avaliar a sexualidade em pacientes pós-acidente vascular encefálico vinculados a unidades de saúde no Pará Métodos: Trata-se de um estudo quanti-qualitativo, descritivo e transversal. Participaram da pesquisa oito pacientes de ambos os sexos, com idade acima de 18 anos, que tiveram Acidente Vascular Encefálico (AVE) isquêmico. Os pacientes foram avaliados quanto a realização e a qualidade do sexo após o AVE de acordo com Índice Internacional de Função Erétil (IIFE) e com o questionário do Quociente Sexual Versão Feminina. Os dados foram organizados em planilhas do Microsoft Excel ${ }^{\circledR} 2016$ e tratados estatisticamente pelo programa estatístico Epi Info 7.2.2. O estudo foi aprovado por Comitê de Ética em Pesquisa Resultados: A média de idade é 57,4 anos, com predominância masculina. Segundo o IIFE, $40 \%$ dos homens foram considerados "normais", com disfunção erétil "leve a moderada" e "moderada", englobaram cada uma, $20 \%$ da amostra, enquanto $20 \%$ não realizavam qualquer forma de atividade sexual. Apenas uma mulher foi submetida ao questionário do Quociente Sexual - Versão Feminina. Esta, teve seu desempenho sexual classificado como "Regular a Bom". Conclusão: Torna-se evidente que o AVE afeta negativamente a qualidade da vida sexual dos pacientes.
\end{abstract}

Palavras-chave: Acidente Vascular Encefálico, Sexualidade, Déficits Neurológicos.

\begin{abstract}
Objective: To determine the sexuality of post-stroke patients linked to health facilities at Pará. Methods: This is a quantitative, qualitative, descriptive and cross-sectional study. Eight patients of both sexes aged over 18 years, who had ischemic stroke, were used. Patients were evaluated for sex performance and quality after stroke according to the International Index of Erectile Function (IIFE) and the Sexual Quotient - Female Version questionnaire. Data were organized in Microsoft Excel 2016 spreadsheets and statistically processed by the Epi Info 7.2.2 statistical program. The Research Ethics Committee Results approved the study: The sample, whose average age is 57.4 years, with male predominance. According to IIFE, $40 \%$ of men were considered "normal", with "mild to moderate" and "moderate" erectile dysfunction, each comprised $20 \%$ of the sample, while $20 \%$ did not perform any form of sexual activity. Only 1 woman was submitted to the Sexual Quotient Female Version questionnaire. This one had its sexual performance classified as "Regular to Good". Conclusion: It is evident that stroke negatively affects the quality of sexual life of patients.
\end{abstract}

Key words: Stroke, Sexuality, Neurological Deficits.

\section{RESUMEN}

Objetivo: determinar la sexualidad de los pacientes con accidente cerebrovascular relacionados con los servicios de salud en el Pará Métodos: Este es un estudio cuantitativo, cualitativo, descriptivo y transversal. Se utilizaron ocho pacientes de ambos sexos, mayores de 18 años, que tuvieron accidente cerebrovascular isquémico. Los pacientes fueron evaluados para el rendimiento sexual y la calidad después del accidente

1Universidade do Estado do Pará (UEPA), Santarém, Pará, Brasil. *E-mail: miguelreboucas1@gmail.com 
cerebrovascular de acuerdo con el Índice Internacional de Función Eréctil (IIFE) y el cuestionario de Cociente Sexual - Versión Femenina. Los datos se organizaron en hojas de cálculo de Microsoft Excel 2016 y se procesaron estadísticamente mediante el programa estadístico Epi Info 7.2.2. El estudio fue aprobado por el Comité de Ética de Investigación Resultados: La muestra, cuya edad promedio es de 57.4 años, con predominio masculino. Según el IIFE, el $40 \%$ de los hombres fueron considerados "normales", con disfunción eréctil "leve a moderada" y "moderada", cada uno de ellos comprendía el $20 \%$ de la muestra, mientras que el $20 \%$ no realizaba ninguna forma de actividad sexual. Solo 1 mujer fue sometida al cuestionario Sexual Cootient - Female Version. Este tenía su desempeño sexual clasificado como "Regular a Bueno". Conclusión: es evidente que el accidente cerebrovascular afecta negativamente la calidad de vida sexual de los pacientes.

Palabras clave: Accidente cerebrovascular, Sexualidad, Déficit neurológico.

\section{INTRODUÇÃO}

Segundo o Instituto Brasileiro de Geografia e Estatística (IBGE) a população brasileira encontra-se em um processo de reestruturação demográfica, apresentando diminuição nas taxas de fecundidade, redução da mortalidade e consequente elevação da expectativa de vida. Sendo assim, está acontecendo um aumento na população idosa brasileira, que, em 2020 , passará a ser $13,7 \%$ da população total e, em 2040 , atingirá o número de 52 milhões, representando praticamente um quarto de todos os habitantes brasileiros. Por consequência, doenças próprias da velhice têm mostrado uma crescente prevalência na sociedade, dentre elas destaca-se o Acidente Vascular Encefálico (AVE) (DUTRA MOM, et al., 2017).

Segundo Romeiro MEG, et al. (2017) o Acidente Vascular Encefálico (AVE) é definido como uma síndrome clínica de desenvolvimento rápido por uma perturbação focal da função cerebral, afetando cerca de 200 mil mortes nos Estados Unidos anualmente. Consiste em um súbito distúrbio vascular que acarreta interrupção de fluxo sanguíneo para tais áreas focais, com mais de 24 horas de duração. Pode-se então, a partir da fisiopatologia, classificar o AVE em acidente vascular encefálico isquêmico (AVEI), onde a interrupção do fluxo sanguíneo é causada por obstrução de uma ou mais artérias por um trombo ou êmbolo, e em acidente vascular encefálico hemorrágico (AVEH), onde a interrupção do fluxo sanguíneo é causado pelo rompimento de uma artéria (DOS SANTOS BBC, et al., 2018).

Um estudo epidemiológico realizado com a população brasileira (Joinville) revelou 105,4 casos/100.000 habitantes/ano, taxa similar às registradas em países industrializados. Faixa etária avançada é o fator de risco de maior peso nas doenças cerebrovasculares: cerca de $75 \%$ dos pacientes com AVE agudo têm idade superior a 65 anos, e a sua incidência praticamente dobra a cada década a partir de 55 anos (MARTINS ERC, et al., 2016).

De acordo com os dados retrospectivos de Brasil (2013) há uma incidência anual de AVE de 108 casos por 100 mil habitantes, uma taxa de fatalidade aos 30 dias de $18,5 \%$ e aos 12 meses de $30,9 \%$, sendo o índice de recorrência após um episódio do AVE de 15,9\%. Dentre os sobreviventes dessa doença, $90 \%$ desenvolvem algum tipo de deficiência ou incapacidade, com prejuízos nas funções sensitivas, motoras, de equilíbrio e de marcha, além do déficit cognitivo, disfágico e de linguagem. Além disso, vão causar em 30 a $40 \%$ dos casos algum tipo de dependência na vida cotidiana, como também consequências psicossociais, tendo contribuição na estigmatização gerada pela sociedade, que pormenoriza àqueles que não possuem plenas capacidades físicas, causando então nesses pacientes maiores chances de adquirirem depressão, ansiedade e distúrbios do sono (VIEIRA DCD, et al., 2017).

Em pacientes que passaram por um AVE, geralmente as respostas e o processamento das excitações sexuais são alterados. A função sexual consiste basicamente em desejo, excitação sexual, orgasmo e resolução, caracterizados pelo relaxamento. Qualquer alteração nesses fatores acaba levando à diminuição da libido, da quantidade de intercursos sexuais, aumento da disfunção erétil e da ejaculação precoce, no caso dos homens, sendo que os AVEs afetam algum desses aspectos. Os relatos são de dor durante o ato sexual ou mesmo um desconforto, o que impacta profundamente a qualidade de vida dessas pessoas, na sua saúde do ponto de vista geral e até mesmo das relações afetivas com seus parceiros, porquanto a sexualidade e o sexo são partes fundamentais do cotidiano humano. (PEREIRA ARR, et al., 2017). 
É importante ressaltar que o tema sobre sexualidade ainda continua sendo um tabu na sociedade, principalmente quando se envolve portadores de deficiência pós-AVE. Isso porque é estipulado perante a sociedade que a sexualidade no idoso é uma forma de perversão, não considerando que o desejo sexual é algo inerente ao ser humano, independente de idade e condição física. Isso vai gerar um fator de inibição da sexualidade desses pacientes, que vão ser pressionados pela sociedade com essa imagem distorcida das suas vidas sexuais, contribuindo, juntamente com as disfunções causadas pela doença, para o abandono da sexualidade (SANTOS MC, 2017).

Segundo Maynard MT, et al. (2017), qualidade de vida é um termo que abrange uma grande gama de significados constituídos por uma diversidade de fatores objetivos (a satisfação com as necessidades básicas e o grau de desenvolvimento socioeconômico) e subjetivos (que são manifestações pessoais, sentimentos e valores) que refletem o conhecimento, as experiências e os valores tanto individuais como coletivos em um contexto cultural, social e histórico. Logo se encaixa o contexto da importância da manutenção dessa qualidade de vida nesses pacientes pós-AVE, pois há uma perda imensurável desta a partir do momento em que essas sequelas afetam desde a satisfação com as necessidades básicas, com os distúrbios em geral, que vão gerar problemas de manifestações pessoais até sensação de corrupção de valores próprios.

Diante disso, este estudo objetiva identificar quais os modos de enfrentamento de um grupo de pacientes com algum tipo de déficit pós AVE estão lidando com essa nova realidade, além de correlacionar com este foco a variável sexualidade, na sociedade santarena.

\section{MÉTODOS}

Tratou-se de um estudo de natureza aplicada, cuja abordagem é quanti-qualitativa e descritivo transversal. Realizado em uma clínica de saúde no Pará mediante autorização da coordenação local. Foram avaliados no setor de fisioterapia 10 indivíduos que tiveram acidente vascular encefálico, regularmente participantes deste programa na época estipulada, foram selecionados de acordo com os critérios de inclusão e exclusão estipulados e compuseram a amostra alcançável do presente estudo. Foram todos contactados por via telefônica e esclarecidos sobre os objetivos da pesquisa e sobre o tema abordado, então, apenas 8 participantes aceitaram participar das entrevistas, sendo então esta a amostra final deste estudo. Os resultados coletados foram armazenados em planilhas do Microsoft Excel® 2016 e foram tratados estatisticamente pelo programa estatístico Epi info® 7.2.2.

Os critérios de inclusão foram baseados em indivíduos com diagnóstico clínico de AVE e que tivessem acima de 18 anos, além de ser o status cognitivo preservado. Para a exclusão, os indivíduos com outra patologia neurológica após o AVE e com dificuldade de compreender comandos verbais não foram analisados. Destaca-se que o trabalho foi aprovado pelo Comitê de Ética em Pesquisa com Seres Humanos da Universidade do Estado do Pará/Campus XII - Santarém, sob CAAE: 01701018.0.0000.5168.

Em relação aos instrumentos de avaliação, foram utilizados dois majoritariamente. O primeiro foi o Índice Internacional de Função Erétil (IIFE) consiste em um instrumento curto e reprodutível para mensuração da função erétil em homens (PEREIRA ARR, et al., 2017).O questionário contém 15 itens e é dividido em cinco domínios da função sexual: função erétil (6 itens), função do orgasmo (2 itens), desejo sexual (2 itens), satisfação no coito (3 itens) e satisfação global (2 itens). Os intervalos da pontuação permitem classificar a gravidade da disfunção sexual (DS) em cinco grupos: Sem DS (26- 30), DS ligeira (22-25), DS ligeira a moderada (17-21), DS moderada (11-16) e DS grave (6-10) Já o segundo, foi o Quociente Sexual - Versão Feminina (QS-F) foi desenvolvido no Programa de Estudos em Sexualidade (PROSEX) do Instituto de Psiquiatria do Hospital das Clínicas da Faculdade de Medicina da Universidade de São Paulo com a finalidade de analisar os vários domínios da atividade sexual feminina de forma simples e objetiva.

No que tange aos instrumentos de avaliação da sexualidade, foram utilizados dois. O primeiro foi o Índice Internacional de Função Erétil (IIFE), que serve para a qualificação da disfunção erétilTal questionário consiste em 15 itens e é dividido em cinco grupos: função erétil, função do orgasmo, desejo sexual, satisfação no coito e satisfação global. O segundo foi o Quociente Sexual - Versão Feminina (QS-F), que tem a finalidade de 
qualificar, também, a atividade sexual sob a ótica feminina. O questionário consiste em dez questão, cada uma com uma escala a ser respondida de 0 a 5 , que se referem, respectivamente ao critério ruim e excelente. As questões norteadoras têm como tema: existência ou não do desejo sexual, preliminares excitação e sintonia com o parceiro, conforto durante a relação e orgasmo e satisfação.

Por fim, tem-se o instrumento de avaliação da qualidade de vida geral dos pacientes pós-AVE. Para isso, foi utilizado a Escala de Qualidade de Vida Específica para AVE (EQVE-AVE), elaborada com 12 domínios de pesquisa que podem ou não ser afetados pelo AVE (DELBONI MCC, et al., 2010). Dentre eles estão energia, papel familiar, linguagem, mobilidade, humor, personalidade, autocuidado, papel social, raciocínio, função de membro superior, visão e trabalho/produtividade, totalizando um total de 49 perguntas divididas entre os temas. O escore segue um valor numérico de 1 a 5 , em escala crescente de positividade e um valor não numérico, sendo considerado entre concordar ou não, ajudar ou não e poder realizar ou não determinada atividade cotidiana.

\section{RESULTADOS E DISCUSSÃO}

Na Tabela 1 pode-se verificar que do total de participantes da pesquisa $(n=8)$ obteve-se como idade média $54,5 \pm 7,4$ anos. No sexo feminino a idade média prevalente foi de $49,7 \pm 2,3$ anos e nos homens foi de 57,4 $\pm 8,1$ anos, compondo uma amostra de adultos jovens acometidos pelo AVC. Sobre a escolaridade, o grupo geral foi dividido equitativamente nos 4 parâmetros possíveis, no entanto, quando analisamos especificamente cada sexo, tem-se que a $66,7 \%$ do sexo feminino $(n=2)$ tem apenas o ensino fundamental completo, enquanto no sexo masculino $40 \%$ tem o ensino superior completo $(n=2)$. Podemos também analisar que o AVE é um fator muito prejudicial ao cotidiano laboral dos pacientes, visto que antes do acontecimento $100 \%$ dos candidatos exerciam uma profissão, enquanto após apenas $12,5 \%$ exerce $(n=1)$.

Tabela 1 - Variáveis epidemiológicas da amostra estudada.

\begin{tabular}{|c|c|c|c|c|c|c|}
\hline \multirow{2}{*}{ Características } & \multicolumn{2}{|c|}{ Feminino $(n=3)$} & \multicolumn{2}{|c|}{ Masculino $(n=5)$} & \multicolumn{2}{|c|}{ Geral $(n=8)$} \\
\hline & $\mathbf{n}$ & $\%$ & $\mathbf{n}$ & $\%$ & $\mathbf{N}$ & $\%$ \\
\hline \multicolumn{7}{|l|}{ Idade } \\
\hline Acima de 55 anos & 0 & $0,0 \%$ & 3 & $60,0 \%$ & 3 & $37,5 \%$ \\
\hline Até 55 anos & 3 & $100,0 \%$ & 2 & $40,0 \%$ & 5 & $62,5 \%$ \\
\hline Média \pm Desvio & \multicolumn{2}{|c|}{$49,7 \pm 2,3$} & \multicolumn{2}{|c|}{$57,4 \pm 8,1$} & \multicolumn{2}{|c|}{$54,5 \pm 7,4$} \\
\hline \multicolumn{7}{|l|}{ Escolaridade } \\
\hline Fundamental incompleto & 0 & $0,0 \%$ & 2 & $40,0 \%$ & 2 & $25,0 \%$ \\
\hline Fundamental Completo & 2 & $66,7 \%$ & 0 & $0,0 \%$ & 2 & $25,0 \%$ \\
\hline Médio Completo & 1 & $33,3 \%$ & 1 & $20,0 \%$ & 2 & $25,0 \%$ \\
\hline Superior completo & 0 & $0,0 \%$ & 2 & $40,0 \%$ & 2 & $25,0 \%$ \\
\hline \multicolumn{7}{|l|}{ Profissão Pré } \\
\hline Sim & 3 & $100,0 \%$ & 5 & $100,0 \%$ & 8 & $100,0 \%$ \\
\hline Não & 0 & $0,0 \%$ & 0 & $0,0 \%$ & 0 & $0,0 \%$ \\
\hline \multicolumn{7}{|l|}{ Profissão Pós } \\
\hline Sim & 1 & $33,3 \%$ & 0 & $0,0 \%$ & 1 & $12,5 \%$ \\
\hline Não & 2 & $66,7 \%$ & 5 & $100,0 \%$ & 7 & $87,5 \%$ \\
\hline \multicolumn{7}{|l|}{ Renda } \\
\hline De 1 a 3 salários & 1 & $33,3 \%$ & 4 & $80,0 \%$ & 5 & $62,5 \%$ \\
\hline De 4 a 6 salários & 1 & $33,3 \%$ & 0 & $0,0 \%$ & 1 & $12,5 \%$ \\
\hline De 7 a 9 salários & 1 & $33,3 \%$ & 0 & $0,0 \%$ & 1 & $12,5 \%$ \\
\hline Acima de 9 salários & 0 & $0,0 \%$ & 1 & $20,0 \%$ & 1 & $12,5 \%$ \\
\hline
\end{tabular}

Fonte: JÚNIOR JJP, et al., 2019

Em relação ao estado civil, $50 \%$ de ambos os sexos eram casados, $100 \%$ possuíam algum grau de escolarização, $100 \%$ se apresentavam com profissão atuante antes do AVC e destes, $87,5 \%$ ficaram sem 
profissão após o AVC, possuindo uma renda de 2 a 3 salários mínimos em $62,5 \%$ dos casos. Discorrendo sobre o gênero, é possível identificar a maior prevalência de indivíduos do sexo masculino, com $62 \%$, seguido pelo sexo feminino, com 38\%. O estudo de Pereira ARR, et al. (2017), realizado com pacientes pós-AVE em uma clínica de reabilitação do Rio Grande do Norte, que teve como objetivo principal analisar a sexualidade pós AVE desses indivíduos, discorda com os dados encontrados na presente pesquisa, pois mostra uma maior prevalência do sexo feminino com $58 \%$ em detrimento do sexo masculino com $42 \%$. Concordando com os resultados desta pesquisa, De Carvalho MIF, et al. (2015), no estado do Ceará, encontrou em seu estudo, feito através da análise de 21 prontuários de pacientes diagnosticados com AVE em um dado hospital, a maior prevalência de homens, representando $52,4 \%$ do total.

De Souza Rodrigues M, et al. (2017) explica que o sexo masculino é um fator de risco imutável para AVE, já que essa população tende a procurar menos ajuda médica e tem menos cuidado com a saúde em comparação com o sexo feminino, e que os fatores de risco modificáveis interferem na influência do sexo na incidência do AVE, como é o caso da hipertensão, que é mais prevalente em homens até a quinta década de vida.

$\mathrm{Na}$ análise da idade, também é possível identificar a maior prevalência de indivíduos de até 55 anos, representando $62,5 \%$. Foi calculada também a média de idade dos entrevistados, que foi de 54,5 anos. Comparando com o estudo de Samson J, et al. (2015), um estudo de coorte realizado em um hospital australiano referência para AVE, que teve como objetivo final entrevistar os pacientes pós AVE atendidos durante o primeiro semestre de 2014 e intervir na qualidade sexual deles através de fisioterapia e psicoterapia, a média de idade dos entrevistados era de 66,3 anos, o que se distancia bastante da média de idade encontrada na presente pesquisa.

Já no estudo de Azanmasso H, et al. (2016), que foi baseado em entrevistas com pacientes pós AVE atendidos no setor de reabilitação do Hospital Universitário Nacional de Benim, mostrou uma média de idade de entrevistados de 54,97 anos, que se aproxima bastante da faixa etária desta pesquisa. $O$ estudo brasileiro de Vasconcelos L, et al. (2017) também corrobora com os dados encontrados nesta pesquisa, pois mostra uma idade média dos indivíduos com AVE estudados de 59,8 anos, mostrado, então, uma idade média menor que 60 anos.

Martins ERC, (2016) explica o motivo da idade menor que 60 anos ser um risco isolado para AVE, quando afirma que o tal risco dobra a cada década em pacientes acima de 55 anos, principalmente devido a alterações cardiovasculares e metabólicas ligadas à idade, tendo como principal exemplo a maior tendência a formação de placas de aterosclerose.

De acordo com dados de 2017 da Organização Mundial da Saúde (OMS), a Austrália ocupa o terceiro lugar na escala mundial de Índice de Desenvolvimento Humano, enquanto o Brasil e Benim estão ambos abaixo da $50^{a}$ posição. Através disso podemos mostrar que faixa etária de indivíduos afetado pelo AVE pode ser influenciado pelas condições socioeconômicas do país, já que o Brasil e Benim possuem um menor desenvolvimento humano quando comparado a Austrália. Quanto ao grau de escolaridade, através dos resultados desta pesquisa não foi visto a prevalência de nenhum grau de escolaridade, tendo apenas como conclusão que todos os participantes eram escolarizados em algum grau.

Comparando com o estudo de Costa FA, et al. (2011), estudo realizado no estado do Rio Grande do Norte com pacientes dos maiores serviços de fisioterapia voltados a reabilitação pós-AVE, vemos que há divergência nos dados encontrados, pois $31,1 \%$ dos participantes eram analfabetos. Já no estudo de Pereira NKF (2018), feito com pacientes atendidos nos ambulatórios de fisioterapia da Universidade Federal do Rio Grande Norte, houve concordância com os achados desta pesquisa, já que $90 \%$ dos pacientes também possuíam algum grau de escolaridade.

De acordo com De Medeiros CSP, et al. (2017) a característica mais comum na sociedade brasileira é de pacientes com baixa escolaridade sendo acometidos pelo AVE, o que contradiz o achado deste estudo, já que a baixa quantidade de informações sobre os meios de prevenção, os hábitos e comportamentos de risco à saúde fazem com que o segmento populacional de baixa escolaridade esteja mais suscetível ao AVE. 
Foi descoberto na atual pesquisa, que $100 \%$ dos participantes tinham uma profissão pré AVE, porém apenas $12,5 \%$ tem emprego após o AVE. Corroborando com esse dado, Damata SRR, et al. (2016) e Barbosa et al. (2017) encontraram que $100 \%$ dos pacientes estudados tinham profissão pré e não tinham profissão pós AVE.

Girão SMM (2015) explica o motivo dessa diminuta quantidade de paciente pós AVE retornarem as suas profissões após a doença, quando diz que graças a sequelas, quer sejam de ordem física e/ou cognitiva, eles ficam, em sua maioria, com sua capacidade funcional reduzida, impedindo com que eles exerçam suas funções trabalhistas prévias.

Quanto a renda familiar, foi visto nesta pesquisa que $65,3 \%$ tinha uma renda de 1 a 3 salários mínimos. Santos NMF e Tavares DMS (2012) também encontraram entre seus resultados uma absoluta prevalência de renda mensal entre 1 a 3 salários mínimos, representando $100 \%$ de sua amostra, concordando com o este estudo. Da mesma forma, Gomes AC, et al. (2012) encontraram em seu estudo feito com 140 pacientes pósAVE em João Pessoa a maior prevalência de renda mensal entre 1 a 3 salários com $49,3 \%$, ratificando o achado da pesquisa apresentada.

A explicação do porquê o AVE ser mais prevalente em indivíduos de baixa renda é bem descrita por De Menezes KVP, et al. (2012) quando diz que populações com níveis socioeconômicos mais baixos estão relacionados ao pouco acesso ao conhecimento e aos serviços de saúde, a um estilo de vida não saudável, à baixa adesão às estratégias de prevenção, inadequadas condições de moradia e aumento do estresse.

A Tabela 2 aborda, ainda, o histórico de doenças concomitantes apresentadas pelos entrevistados no momento de ocorrência do acidente vascular encefálico. Como observado nesta tabela, o Diabetes Mellitus isolado figurou como a principal comorbidade prévia dentre os pacientes que evoluíram com AVE inclusos nesta pesquisa, contando com $60 \%$ dos entrevistados $(n=4)$. Importante causa de eventos hemorrágicos, os aneurismas de vasos intracranianos figuraram como segunda condição mais frequente, observados em $25 \%$ dos entrevistados ( $n=2$ ), sendo todos do sexo feminino - acometendo $66,7 \%$ das mulheres deste estudo. Hipertensão Arterial isolada e a associação desta com o diabetes mellitus foram observados, cada uma, em 1 entrevistado, atingindo uma porcentagem individual de $12,5 \%$.

Tabela 2 - Variáveis clínicas da amostra estudada.

\begin{tabular}{lllllll}
\hline \multirow{2}{*}{ Características } & \multicolumn{2}{c}{ Feminino $\mathbf{( n = 3 )}$} & \multicolumn{2}{c}{ Masculino $(\mathbf{n}=\mathbf{5})$} & \multicolumn{2}{c}{ Geral $(\mathbf{n}=\mathbf{8})$} \\
\cline { 2 - 7 } & $\mathbf{n}$ & $\%$ & $\mathbf{n}$ & $\%$ & $\mathbf{n}$ & $\%$ \\
\hline Tipo de AVE & & & & & & \\
\hline Hemorrágico & 2 & $66,7 \%$ & 0 & $0,0 \%$ & 2 & $25,0 \%$ \\
Isquêmico & 1 & $33,3 \%$ & 5 & $100,0 \%$ & 6 & $75,0 \%$ \\
\hline Históricos de doenças & & & & & & \\
\hline Aneurisma & 2 & $66,7 \%$ & 0 & $0,0 \%$ & 2 & $25,0 \%$ \\
Diabetes & 1 & $33,3 \%$ & 3 & $60,0 \%$ & 4 & $50,0 \%$ \\
Hipertensão arterial & 0 & $0,0 \%$ & 1 & $20,0 \%$ & 1 & $12,5 \%$ \\
Diabetes+ Hipertensão arterial & 0 & $0,0 \%$ & 1 & $20,0 \%$ & 1 & $12,5 \%$ \\
\hline
\end{tabular}

Fonte: JÚNIOR JJP, et al., 2019.

Rodrigues ESR, et al. (2013) aponta que o AVE é, na maioria dos casos, secundário a eventos cardiovasculares preveníveis, que aumentam o risco de desenvolver este quadro. Neste âmbito, Carvalho MA et al. (2015) aponta a hipertensão arterial sistêmica (HAS), o diabetes mellitus (DM), o tabagismo, as dislipidemias, o sedentarismo e a fibrilação atrial como fatores de risco descritos como modificáveis, e que estão diretamente ligados ao estilo de vida de cada pessoa. Gouvêa D, et al. (2015) aponta o aneurisma como importante causa de acidente vascular encefálico hemorrágico.

Em comparação com outros trabalhos, retificou-se a importância destas doenças na gênese do AVE, porém, com certa divergência em relação as suas ordens de prevalência. Tanto Carvalho MA, et al. (2015), 
quanto Rodrigues ESR, et al. (2013) apontaram a hipertensão arterial sistêmica como doença pregressa de maior prevalência dentre os indivíduos que evoluíram com AVE em suas amostras. Estes estudos, apontaram, respectivamente, que $68,8 \%$ e $30,8 \%$ apresentavam a doença. A prevalência de Diabetes Mellitus nestas pesquisas foi $19,7 \%$ e $20,3 \%$, respectivamente, o que colocou esta patologia como terceira doença prévia mais prevalente nestes estudos. Ambas as pesquisas não mencionaram a prevalência dos Aneurismas em seus trabalhos.

Independentemente de sua ordem, é consenso que as patologias expostas, com exceção dos aneurismas, se comportam como fatores preveníeis e figuram como relevantes patologias relacionadas a eventos cerebrovasculares. Deste modo, estas se apresentam como importantes alvos de saúde pública e individual na prevenção destes eventos.

Em relação à qualidade de vida, mensurada pelo EQVE-AVE e analisada através da Tabela 3, observamos que a pontuação geral da amostra foi de $168,0 \pm 43,6$ que equivale a um percentual de $68 \%$ de QV, já que a pontuação geral ideal seria de 245 pontos que equivale a 100\% percepção de QV. Quando distribuímos em relação aos sexos, observamos que os homens possuem uma melhor percepção da QV (73\%) quando comparados às mulheres com AVC $(60 \% Q V)$. Dos entrevistados, apenas $25 \%(n=2)$ foram classificados como baixa Qualidade de Vida pelo EQVE-AVE (escore total <147), sendo um do sexo masculino e um do feminino, ambos com menos de 60 anos de idade.

Tabela 3 - Qualidade de vida da amostra estudada.

\begin{tabular}{|c|c|c|c|}
\hline Qualidade de vida & Feminino $(n=3)$ & Masculino $(n=5)$ & Geral $(n=8)$ \\
\hline Energia & $2,7 \pm 0,3$ & $3,3 \pm 1,7$ & $3,1 \pm 1,4$ \\
\hline Papeis familiares & $2,6 \pm 1,3$ & $3,4 \pm 2,2$ & $3,1 \pm 1,8$ \\
\hline Linguagem & $3,1 \pm 1,7$ & $4,7 \pm 0,3$ & $4,1 \pm 1,2$ \\
\hline Mobilidade & $3,5 \pm 0,9$ & $3,7 \pm 1,0$ & $3,6 \pm 0,9$ \\
\hline Humor & $3,1 \pm 1,3$ & $4,1 \pm 1,2$ & $3,7 \pm 1,2$ \\
\hline Personalidade & $2,7 \pm 2,1$ & $3,3 \pm 1,5$ & $3,1 \pm 1,6$ \\
\hline Autocuidado & $3,8 \pm 0,7$ & $3,7 \pm 1,2$ & $3,8 \pm 1,0$ \\
\hline Papeis sociais & $1,4 \pm 0,4$ & $2,6 \pm 1,8$ & $2,2 \pm 1,5$ \\
\hline Memória & $2,6 \pm 0,8$ & $4,3 \pm 0,8$ & $3,7 \pm 1,2$ \\
\hline Função MMSS & $3,5 \pm 1,1$ & $3,6 \pm 1,1$ & $3,6 \pm 1,0$ \\
\hline Visão & $3,7 \pm 2,0$ & $4,2 \pm 1,3$ & $4,0 \pm 1,5$ \\
\hline Trabalho & $3,0 \pm 0,0$ & $3,0 \pm 1,4$ & $3,0 \pm 1,0$ \\
\hline Geral & $147,0 \pm 30,3$ & $180,6 \pm 48,4$ & $168,0 \pm 43,6$ \\
\hline
\end{tabular}

Fonte: JÚNIOR JJP, et al., 2019

Em geral, houve maior pontuação na EQVE-AVE em relação neste estudo do que em trabalhos como o de Rangel ESS, et al. (2013), que avaliou 139 indivíduos e obteve uma pontuação média geral de 139,7 \pm 38,4. Em relação a outros trabalhos, Moreira NRTL, et al. (2015) demonstrou dados relativamente semelhantes, apontando que 30,3\% de sua amostra apresentava baixa QV após 6 meses do AVE.

A Tabela 3 demonstra ainda, os domínios avaliados pela escala EQVE-AVE. Tanto no geral como no sexo masculino, as áreas com menor pontuação foram "papeis sociais" e "trabalho" e as de maior pontuação "linguagem". No sexo feminino, "papeis sociais" recebeu a pontuação mais baixa, enquanto "autocuidado" a mais alta.

Em comparação, houve divergência em relação ao estudo de Rangel ESS et al. (2013), que apontou como domínios mais comprometidos a "mobilidade", "trabalho" e "função do membro superior". Já em relação à pesquisa de Moreira NRTL, et al. (2015) observou-se certa semelhança neste aspecto. Neste, os domínios mais comprometidos foram "energia", "papeis sociais", "papeis familiares" e "personalidade". 
Dentre as áreas mais afetadas neste trabalho, está a relevância observada na modificação dos papeis sociais do indivíduo o que sugere a relevante influência negativa que o acidente vascular encefálico exerce na reintegração dos afetados à sociedade. Esta análise demonstra um importante ponto que necessita de melhor intervenção, com ênfase no acolhimento destas pessoas nas comunidades em que participam e no estímulo ao convívio social com os entes que previamente se relacionavam com os mesmos.

Outro ponto importante refere-se ao comprometimento do "trabalho", que pode estar relacionado com o dado já descrito de que grande parte da amostra acabou por abandonar seus ofícios após o AVE. Este fator, além de exercer impacto individual nas finanças do doente e das famílias afetadas, pode afetar de forma mais ampla a própria sensação de capacidade do indivíduo.

Quanto a sexualidade, verificada na Tabela 4, observou-se que dos 8 indivíduos inclusos na pesquisa, 3 relataram não ter realizado qualquer tentativa de relação sexual após o episódio de AVE, demonstrando um comprometimento maior deste critério tão importante para a qualidade de vida. Este fato impossibilitou a aplicação dos instrumentos relativos a sexualidade a estes entrevistados, que foram inclusos na tabela como "Não aplicável".

Tabela 4 - Perfil da Sexualidade dos participantes.

\begin{tabular}{llll}
\hline Disfunção Sexual & \multicolumn{2}{l}{ Sexo } & Geral \\
\hline Disfunção Erétil & \multicolumn{2}{l}{ Masculino (n=5) } & Masculino \\
\cline { 2 - 4 } & $\mathbf{N}$ & $\%$ & \\
\hline Normal & 2 & $40 \%$ & $25 \%$ \\
\hline Leve a moderada & 1 & $20 \%$ & $12,5 \%$ \\
\hline Moderada & 1 & $20 \%$ & $12,5 \%$ \\
\hline Não aplicável & 1 & $20 \%$ & $12,5 \%$ \\
\hline Total (Homens) & 5 & $20 \%$ & $62,5 \%$ \\
\hline Quociente Sexual (Sexo Feminino) & \multicolumn{1}{l}{ Feminino $(\mathbf{n}=\mathbf{3})$} & & Feminino \\
\cline { 2 - 4 } & $\mathbf{N}$ & $\%$ & $12,5 \%$ \\
\hline Regular a Bom & 1 & $33 \%$ & $25 \%$ \\
\hline Não aplicável & 2 & $67 \%$ & $37,5 \%$ \\
\hline Total (Mulheres) & 3 & $100 \%$ & $100 \%$ \\
\hline Total & 8 & $100 \%$ & \\
\hline
\end{tabular}

Fonte: JÚNIOR JJP, et al., 2019

Em relação aos indivíduos do sexo masculino submetidos ao questionário proposto no Índice Internacional de Função Erétil (IIFE), 40\% ( $n=2)$ foram classificados como "normais" em relação a este critério. Disfunção erétil "leve a moderada" e "moderada", englobaram cada uma, 1 indivíduo, ou $20 \%$ da amostra, evidenciando que $40 \%$ dos homens deste estudo demonstravam objetivamente algum grau de disfunção erétil, enquanto $20 \%(n=1)$ não realizavam qualquer forma de atividade sexual.

Das 3 mulheres entrevistadas, apenas 1 foi submetida ao questionário do Quociente Sexual - Versão Feminina. Esta teve seu desempenho sexual classificado como "Regular a Bom" segundo este instrumento e equivale, relativamente, a $33 \%$ dos indivíduos do sexo feminino ou à $12,5 \%$ do total da amostra. As demais mulheres entrevistadas não empreenderam qualquer tentativa de realização de atividade sexual após o acidente vascular cerebral e, como os indivíduos do sexo masculino previamente citados, foram caracterizadas como "Não Aplicável" para este critério na tabela acima.

Como apontado nesta pesquisa, uma revisão literária realizada por Monteiro ES, et al. (2012), aponta a diminuição da função sexual é comum após o AVE, muitas vezes por temerem ter uma vida ativa após o AVC, por uma limitação propriamente dita da função sexual ou por outros problemas físicos e/ou funcionais.

Assim como nesta pesquisa, Giaquinto $S$, et al. (2003) apontou, em seu estudo, que também houve número considerável de indivíduos que não mais realizaram atividade sexual após o AVE, representados em 
$50 \%$ de sua amostra, achado que predominou - assim como nesta pesquisa - no sexo feminino. Pereira ARR, et al. (2017) também apresentou dados semelhantes, com apenas $48,3 \%$ de sua amostra caracterizados como sexualmente ativos. As motivações por trás desse achado - da não realização de uma vida sexual ativa - também foram evidenciadas por esta fonte, que apontou como causas a perda do interesse, o medo e a falta de parceiros após o AVE.

Quanto à avaliação objetiva da função sexual, realizados por meio dos instrumentos IIFE e QS-F, Pereira ARR, et al. (2017) observou resultados diferentes, apontando que $77,7 \%$ dos homens de sua pesquisa apresentavam algum grau de disfunção erétil - sendo esta mínima em $71,4 \%$-, frente a $40 \%$ dos homens desta pesquisa. Estes autores observaram em seu trabalho que $40 \%$ das mulheres alcançaram função sexual satisfatória (com mais de 60 pontos no QS-F), frente a 33\% das mulheres deste estudo.

Quanto a correlação da CIF com a EQVE-AVE, na Tabela 5, no item energia, 50\% da amostra relatou na resposta 1 se sentir cansado a maior parte do tempo ou resposta 4 (estar cansado demais para fazer o que queria). No item papeis familiares, observamos que em relação às atividades de recreação e lazer (D920) e nas atitudes individuais de familiares próximos (E410), 50\% relataram que a condição física interferiu nestas situações com os familiares. Interferência esta, no item D920, também observada por Maynard MT, et al. (2017). Já Pereira JMV (2016), demonstrou em seu trabalho menor influência do status físico nesta área.

Em relação à linguagem, 75\% dos pacientes não relataram importante interferência relacionada ao código D3600 da CIF, que corresponde a utilização de dispositivos para comunicação, equivalendo ao questionamento 5 ("Você teve que repetir para que os outros pudessem te entender?") do EQVE-AVE. Em comparação indireta, Maynard MT, et al. (2017) apontou que 41,7\% de sua amostra demonstrou algum grau de influência negativa nesta área.

\section{CONCLUSÃO}

Desta forma, torna-se evidente a necessidade de ação sobre situações estabelecidas antes e após a ocorrência do AVE. Neste quesito, é importante a intervenção sobre diversos fatores de risco para o evento cerebrovascular, por meio da prevenção e controle dos fatores de risco modificáveis e promoção de hábitos e do envelhecimento saudável. Além disso, frente a complexa influência exercida por esta patologia na funcionalidade e na vida privada e coletiva do doente - desde a atividades cotidianas e relações sexuais, até o trabalho e as relações sociais -, torna-se, também, indispensável, a realização de ações que proporcionem uma correta reintegração, não apenas física, mas social e sexual de todos os envolvidos.

\section{REFERÊNCIAS}

1. AZANMASSO H, et al. Sexual disorders in stroke patients compared with control subjects in Benin: A prospective study. Annals of physical and rehabilitation medicine, v. 59, p. e69-e70, 2016.

2. BARBOSA RA, et al. Perfil dos pacientes adultos com acidente vascular encefálico tratados em uma clínica-escola de fisioterapia. Revista de Atenção à Saúde (antiga Rev. Bras. Ciên. Saúde), v. 15, n. 51, p. 5-10, 2017.

3. BRASIL, Ministério da Saúde. Diretrizes de atenção à reabilitação da pessoa com acidente vascular cerebral. Brasília, DF: Secretária de Atenção à Saúde; 2013.

4. CARVALHO MA, et al. Epidemiologia dos acidentes vasculares encefálicos atendidos por meio do serviço de atendimento móvel de urgência. Revista de Enfermagem UFPE on line, Recife, v. 9, n. 3, p. 1015-1021, 2015.

5. DAMATA SRR, et al. Perfil epidemiológico dos idosos acometidos por acidente vascular cerebral. Revista Interdisciplinar, v. 9, n. 1, p. 107-117, 2016.

6. DE CARVALHO MIF, et al. Acidente vascular cerebral: dados clínicos e epidemiológicos de uma clínica de fisioterapia do sertão nordestino brasileiro. Revista Interfaces: Saúde, Humanas e Tecnologia, v. 2, n. 6, 2015.

7. DE MEDEIROS CSP, et al. Perfil social e funcional dos usuários da estratégia saúde da família com acidente vascular encefálico. Revista brasileira de ciências da saúde, v. 21, n. 3, p. 211-220, 2017.

8. DE MENESES KVP, et al. Perfil sócio demográfico e áreas de desempenho ocupacional afetadas em pacientes pósAVE atendidos por um serviço de terapia ocupacional. Revista de Terapia Ocupacional da Universidade de São 
Paulo, v. 23, n. 2, p. 107, 2012.

9. DE SOUSA RODRIGUES $\mathrm{M}$, et al. Fatores de risco modificáveis e não modificáveis do AVC isquêmico: uma abordagem descritiva. Revista de Medicina, v. 96, n. 3, p. 187-192, 2017.

10. DELBONI MCC, et al. Relação entre os aspectos das alterações funcionais e seu impacto na qualidade de vida das pessoas com sequelas de Acidente Vascular Encefálico (AVE). O Mundo da Saúde, v.34, n.2, p.165-175, 2010.

11. DOS SANTOS BBC, et al. Perfil Epidemiológico Dos Óbitos Por Acidente Vascular Encefálico No Estado De Mato Grosso Em 2014. In: Anais do Congresso Regional de Emergências Médicas (CREMED-CO), 2018.

12. DUTRA MOM, et al. Revista Brasileira de Epidemiologia. Revista Brasileira de Epidemiologia, v. 20, p.124-135, 2017.

13. GIAQUINTO S, BUZZELLI S, FRANCESCO LD. Evaluation of sexual changes after stroke. J Clin Psychiatry 2003; 64:302-7.

14. GIRÃO SMM. Determinantes da incapacidade funcional em doentes com AVC. 2015. Tese de Doutorado.

15. GOMES AC, et al. Perfil dos usuários pós-acidente vascular cerebrais adscritos à saúde da família no município de João Pessoa. In: 10 Congresso Internacional da Rede Unida. 2012.

16. GOUVÊA D, et al. ACIDENTE VASCULAR ENCEFÁLICO: UMA REVISÃO DA LITERATURA. Ciência Atual-Revista Científica Multidisciplinar das Faculdades São José, v. 6, n. 2, 2015.

17. KASPER DI, et al. Harrison Medicina Interna. 19. ed. Rio de Janeiro: McGraw Hill, 2016.

18. MARTINS ERC, et al. Estudo epidemiológico sobre acidente vascular encefálico em uma clínica escola de Fisioterapia. Revista de saúde pública do Paraná, v. 17, n. 1, p. 32-38, 2016.

19. MAYNARD MT, et al. Linguagem e funcionalidade de adultos pós-Acidente Vascular Encefálico (AVE): avaliação baseada na Classificação Internacional de Funcionalidade, Incapacidade e Saúde (CIF). In: CoDAS. Sociedade Brasileira de Fonoaudiologia, 2017.

20. MONTEIRO ES, et al. Disfunções Sexuais em Pacientes Após Acidente Vascular Cerebral. Rev Neurocienc, v.20, n.3, p.462-467, 2012.

21. MOREIRA NRTL, et al. Qualidade de vida em indivíduos acometidos por Acidente Vascular Cerebral. Rev Neurocienc, v. 23, n. 4, p. 530-537, 2015.

22. PEREIRA ARR, et al. Associação entre função sexual, independência funcional e qualidade de vida em pacientes após acidente vascular encefálico. Revista Fisioterapia e Pesquisa, v. 24, n. 1, p. 54-61, 2017.

23. PEREIRA JMV. Proposta de Core Set para avaliação da capacidade funcional e cognitiva em indivíduos acometidos por AVC em fase crônica. 2016.

24. PEREIRA NKF. Concordância intra e inter-examinador da Theory of Mind Task Battery (ToM) para pacientes pós-Acidente Vascular Cerebral (AVC). 2018. Trabalho de Conclusão de Curso. Universidade Federal do Rio Grande do Norte.

25. RANGEL ESS; BELASCO AGC; DICCINI S. Qualidade de vida de pacientes com acidente vascular cerebral em reabilitação. Acta Paulista de Enfermagem, 2013.

26. RODRIGUES ESR, et al. Fatores de risco cardiovascular em pacientes com acidente vascular cerebral. Amazônia: Science \& Health, v. 1, n. 2, p. 21-28, 2013.

27. ROMEIRO MEG; GUIMARÃES RN; GOMES G. Os Benefícios na Utilização da Terapia Contenção Induzida em Pacientes Idosos com Acidente Vascular Encefálico (AVE). Rev Esc Enf USP, v.12, 2017.

28. SANSOM J, et al. Let's talk about sex: A pilot randomised controlled trial of a structured sexual rehabilitation programme in an Australian stroke cohort. International Journal of Therapy and Rehabilitation, v. 22, n. 1, p. 2129, 2015.

29. SANTOS MC, et al. Percepções e vivências de idosos sobre sua sexualidade. Almanaque multidisciplinar de pesquisa, v.1, n.1, 2017.

30. SANTOS NMF, TAVARES DMS. Correlação entre qualidade de vida e morbidade do cuidador de idoso com acidente vascular encefálico. Rev Esc Enferm USP, v.46, n.4, p.960-6, 2012.

31. SILVA EL, MENEZES EM. Metodologia da pesquisa e elaboração de dissertação. 4. ed. Rev. Atual, $138 p, 2005$.

32. VASCONCELOS $L$, et al. Perfil dos indivíduos com alterações funcionais características de heminegligência após avc. Revista Pesquisa em Fisioterapia, v. 7, n. 2, p. 244-254, 2017.

33. VIEIRA DCD, et al. Reabilitação de acidente vascular encefálico: revisão de literatura. Revista de Atenção à Saúde (antiga Rev. Bras. Ciên. Saúde), v. 15, n. 52, p. 89-95, 2017. 